\title{
Experimental Investigation on Vibration Reduction Performance of Fiber Metal Laminate Beams with MRE Core
}

\author{
Jintong HAN ${ }^{1}$, Wenyu WANG ${ }^{1,2}$, Xintong WANG ${ }^{2}$, Xiaoyue $\mathrm{HU}^{2}$, Ziheng WANG ${ }^{2}$, Zhijiang GAO ${ }^{2}$, Hui LI ${ }^{2 \star}$
}

1 Northeast Yucai Foreign Language School, Shenyang, Liaoning, China, 110819;

2 School of Mechanical Engineering \& Automation, Northeastern University, Shenyang, Liaoning, China, 110819

*Corresponding Author: Hui LI; Wenhua Road No. 11, Northeastern University, Shenyang, Liaoning, 110819, China; Ih200300206@163. com

\begin{abstract}
:
The vibration reduction characteristics of composite beams filled with magnetorheological elastomer core are studied experimentally. The fiber metal laminates with magnetorheological elastomers core is self-designed and prepared. Internal magnetic field is applied to the beam to explore its action of damping vibration performance under the magnetic field for the first time. The composite elements test system with controllable magnetic field intensity is designed and the function of each part is introduced. Then, a set of reasonable and standard vibration test flow of this type of composite beam under different magnetic field intensity is clarified, and the practical test is conducted. It has been found that the composite beam has excellent damping performance with the first 4 damping ratios being greater than $10 \%$. Moreover, after the magnetic field is applied, its damping results can be further improved to meet the active control purpose.

Keywords: magnetorheological elastomer; fiber metal hybrid composite beam; magnetic field intensity; vibration characteristics; damping ratio
\end{abstract}

\section{Introduction}

Fiber metal laminate (FML) is a new type of non-homogeneous composite materials that combine the superior ductility and corrosion resistance of metal materials, and the advantage of lightweight and high strength of fiber-reinforced composite materials ${ }^{[1-3]}$. Nowadays, there are an increasing number of FML plates being used in the fuselage skin, vertical and horizontal tail leading edge, rectifying plate, fairing, upper fuselage siding and the upper slab of the military and civil aircraft ${ }^{[4-5]}$. Damping is an important indicator of the dynamic properties of this type of materials and structures, which is very critical to anti-vibration, shock resistance and noise control ${ }^{[6-8]}$. Magnetorheological Elastomer (MRE) is an upgraded version of the magnetically controlled fluid magnetorheological fluid (MRF) material, which overcomes the shortcomings of easy settlement and poor stability of $M R F{ }^{[9-11]}$. If the adjustable damping properties of the MRE can be effectively utilized, the vibration resistance, stability and fatigue durability of the whole laminate structure can be greatly improved.

At present, some researchers have carried out lots of researches on the vibration control problems of metal, composite beam, plate and shell structures with MRE core. Choi et al. ${ }^{[12]}$ studied the vibration response to metal beams filled with an MRE core, and found that with the increase in thickness of the MRE core, the structural damping performance tends to rise. Dwivedy et al. ${ }^{[13]}$ used the finite element method to analyze the vibration and stability of metal beam structures with an MRE core under various boundary conditions, and verified the performance advantages of this type of structure in the active vibration control. $\mathrm{Ni}$ et al. ${ }^{[14]}$ studied the vibration response of a metal sandwich beam filled with an MRE core under random excitation, and found that the change of the external magnetic field has an important influence on the shear modulus of the structure. Deng et al ${ }^{[15]}$ developed an adaptive tuning vibration absorber (ATVA) based on MRE. Through test comparison, it was found that ATVA was superior to the traditional passive damper in terms of vibration absorption capacity. Wei et al. ${ }^{[16]}$ studied the vibration control of a metal sandwich beam filled with an MRE core, and found that in different excitation frequency ranges, the control effect of the applied magnetic field was quite different. Usually, the closer to the natural frequency, the better it will be for the vibration control effect of such 
beam structure. Hu et al. ${ }^{[17]}$ studied the suppression effect of the vibration response to metal sandwich beams filled with MRE and MRF materials. It was found that their natural frequencies decreased with the increase in the applied magnetic field intensity, and the MRE has better vibration damping performance than that of the MRF.

The literature survey of the work presented here proves to be limited research work on MRE material applied in the field of active vibration control of FML composite materials, especially with the lack of systematic analysis and solution of structural damping characteristics. Therefore, from the perspective of integrated design of structure and function, the fiber metal laminates with an MRE core (MRE-FML) are developed in this paper. Further, by taking the beam structure with MRE core as an example, its vibration reduction performances under different magnetic field intensities are investigated and evaluated based on the experimental results.

\section{Preparation of the MRE-FML beam}

The studied MRE-FML beam is composed of two metal layers, two fiber-reinforced layers and an MRE core, whose structural schematic is shown in Fig. 1.

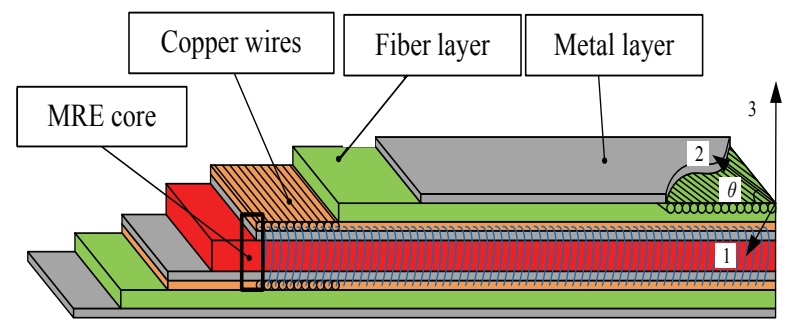

Figure1. Theoretical model of MRE-FML composite beam

Silicone rubber and silicone oil were used as the matrix materials, and carbonyl iron powders were used as the ferromagnetic particles of the MRE. The mass ratio between carbonyl iron powders, silicone rubber and silicone oil is $7: 1.5: 1.5$. During the preparation process, firstly, weigh a certain amount of carbonyl iron powders in a beaker using an electronic scale. After adding the corresponding proportion of silicone oil and rubber materials, stir the mixture with a glass rod until it is even, and then let it stand for 30 minutes to remove bubbles (the time should not be too long to avoid solidification). Next, an aluminum alloy beam was placed in a specific mold, and the viscous mixture of the uncured MRE was evenly spread on the surface of aluminum alloy beam. Subsequently, another aluminum alloy beam is symmetrically covered on the uncured MRE. Then, this laminated structure was repeatedly rolled using a press roll and molded at room temperature for 24 hours. Afterwards, the copper coils were wound on the both surface to the sandwich structure to finish the fabrication of the MRE core. Noted that it already includes the two layers of coils, two layers of aluminum alloy and one layer of MRE material. The detailed material composition of MRE core is listed in Table 1.
Table 1. MRE core material composition

\begin{tabular}{cccc}
\hline Order & Type & mass/g & $\begin{array}{c}\text { thickness/ } \\
\mathrm{mm}\end{array}$ \\
\hline 1 & aluminum alloy(each layer) & 6 & 0.3 \\
2 & copper coil & 3 & 0.3 \\
3 & carbonyl iron powder & 28 & \\
4 & silicone oil & 6 & 2 \\
5 & silicone rubber & 6 & \\
\hline
\end{tabular}

After the MRE core materials were fabricated, the unidirectional carbon-fiber fabrics and epoxy resins were laid to form the fiber-reinforced layers (whose laying information is $\left.\left[\left(0^{\circ} / 90^{\circ}\right)_{3}\right]\right)$ on their upper and lower sides, and the two layers of aluminum alloy were laid on the outside of the used fiber layers. Finally, press this laminated structure tightly with a tempered glass plate to remove bubbles (which needs to be done by repeated rolling with a foam roller). When the whole structure was fully cured, its edge burrs need to be removed by a table saw to obtain the standard MRE-FML beam specimen. Only in this way, the prepared MRE-FML beam can meet the test requirements in the experiment.

\section{Vibration reduction performance test of the MRE-FML beam specimen}

\subsection{Test objects and system}

In this paper, the MRE-FML beam prepared in Section 1 is considered as the research object. Firstly, a clamping fixture, as shown in Fig. 2, is used to clamp one of the short edge. The length, width and thickness of the beam under restraint are $200 \times 30 \times 5 \mathrm{~mm}$ (the clamping length is $40 \mathrm{~mm}$ ).

Fig. 2 shows the developed vibration test system of the MRE-FML beam considering the internal magnetic field effect. By adjusting this internal magnetic field intensity, the damping performance of MRE-FML beam can be quantitatively controlled. The vibration test system mainly includes: (1) PCB086C01 modal force hammer; (2) BK4517 lightweight accelerometer; (3) LMS SCADAS 16-channel data acquisition; (4) DELL M4800 laptop workstation; (5). $24 \mathrm{~V}$ DC power supply and $2 \Omega$ resistance.

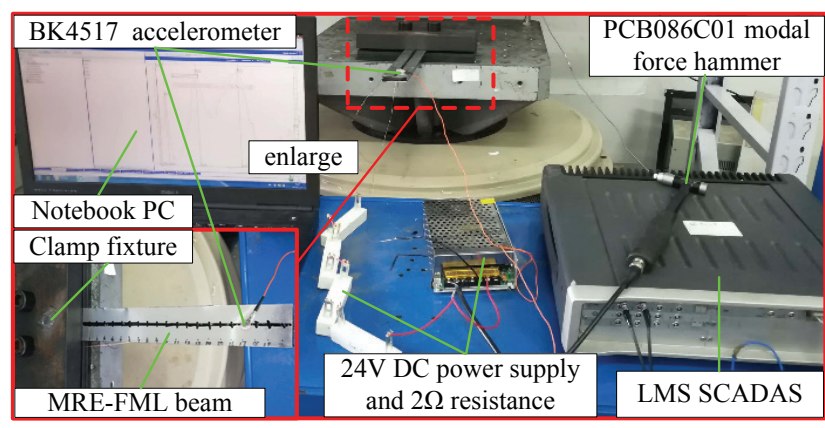

Figure 2 Vibration test system of MRE-FML beam under magnetic field 


\subsection{Test procedure the MRE-FML beam}

The detailed test procedures are summarized as follows:

(1) Connect the experimental circuit to ensure the test reliability of all connected parts

Firstly, connect the copper wires in the MRE-FML beam to the circuit, and turn on the circuit switch to adjust the resistance to an appropriate value. Noted that this step is necessary before the formal experiment, which can ensure the test stability and reliability of all connected sensors and instruments.

(2) Test the natural frequency and damping characteristics of the MRE-FML beam without the magnetic field

Employ the PCB086C01 modal force hammer and BK4517 lightweight accelerometer to conduct the modal experiment on the MRE-FML beam without the magnetic field, where the excitation and response signals are acquired in real time by the LMS SCADAS 16-channel data acquisition. Then, based on the experimental frequency response function data, the first 4 natural frequencies and modal damping ratios can be obtained by PolyMAX method in LMS Test.Lab 14A software.

(3) Test the natural frequency and the damping performance of MRE-FML beam after the magnetic field is applied

After the current in the copper wires of MRE-FML

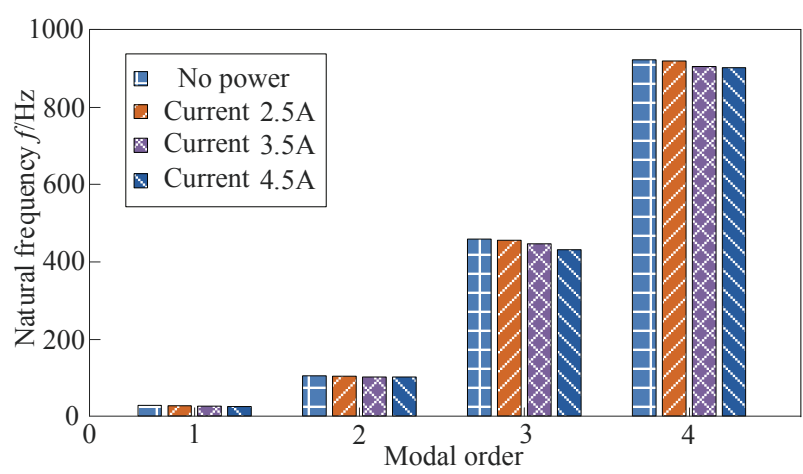

Figure 3. The first four natural frequencies under different magnetic field conditions

Analysis of the above results shows that:

(1) After the magnetic field is applied, the natural frequencies of all orders of the MRE-FML beam show a trend of decrease. The natural frequencies of the third and fourth orders decrease by $2 \%$ to $5 \%$, while the natural frequencies of the first and second orders do not change significantly. This may be because the magnetorheological effect is relatively small under low magnetic field intensity, which makes the change of low order natural frequency of laminated beams not obvious.

(2) The MRE-FML beam has excellent passive damping performance with the first 4 damping ratios being greater than $10 \%$, which is significantly greater than the general damping behavior of composite structure, such as fiber reinforced or fiber-metal laminates (which is usually $1-3 \%)$. Moreover, after the magnetic field is applied, its beam is kept on 1 minute (to let the deformation of MRE core reach a stable state), the modal experiment on the MRE-FML beam with the magnetic field (this time with the current of $2.5 \mathrm{~A}$ ) is carried out again. Then, the first 4 natural frequencies and modal damping ratios can be obtained by using the same test techniques.

(4) Test the natural frequency and damping performance of MRE-FML beam under different magnetic field conditions

Set other two different currents in the copper wires of MRE-FML beam, such as 3.5A and 4.5A, and measure the first 4 natural frequencies and modal damping ratios with the same excitation position and the response position used in the step(3). Then, the effect of vibration reduction can be compared and evaluated under different magnetic field conditions.

\section{Experimental investigation of vibration re- duction performance of MRE-FML beam}

Here, the influence of different magnetic field conditions on the natural frequencies and damping behavior of the MRE-FML beam is investigated, which is based on a series of experimental results (whose test procedure has already been described in Section 3.2). Fig. 3 and Fig. 4 show the first 4 natural frequencies and modal damping ratios.

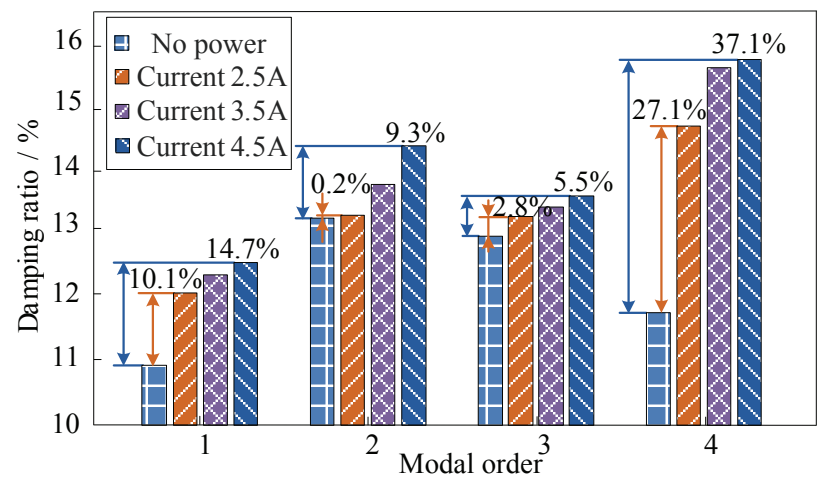

Figure 4. The first four damping ratios under different magnetic field conditions

damping results can be further improved. For example, the increased degree of first four damping ratios reach to $14.7,9.3,5.5$ and $37.1 \%$, respectively when the current is raised to $4.5 \mathrm{~A}$. This proves the feasibility that continuously increasing magnetic field intensity (which is proportional to current) can facilitate the improvement of active damping performance. This increased active damping performance is due to the raised loss factor of MRE materials when the applied magnetic field intensity is increased. Also, this means that the damping performance of the MRE-FML beam can be accurately controlled, which can facilitate to achieve the goal of the quantified control of structural vibration response. However, it should be noted that since the external magnetic field is generated by the current which will lead to an increase in the temperature of the winding copper coil, in the following studies, it is necessary 
to quantitatively evaluate the influence of temperature variation on the damping performance.

\section{Conclusion}

In this paper, by taking the beam structure as an example, MRE-FML structural functional integration materials are prepared, and relevant test system is established to study its damping and vibration reduction performance. It has been found that:

(1) MRE-FML has excellent damping performance, which can be further improved after the magnetic field is applied. Especially, the fourth order damping was increased by $35 \%$.

(2) The damping performance of the MRE-FML beam can be precisely controlled by changing the magnetic field intensity.

Therefore, the vibration response can be precisely controlled. In the future research, it is necessary to carry out more theoretical and experimental studies, especially in active vibration control strategies and methods.

Acknowledgements: This study was supported by the National Natural Science Foundation of China (granted No. 51505070), the Fundamental Research Funds for the Central Universities of China (granted No. N160313002, N160312001, N170302001, N180302004, N180703018, N180312012 and N180313006), the National Key Laboratory of Science and Technology on Advanced Composites in Special Environments, Harbin Institute of Technology (granted No. 6142905192512).

\section{References}

[1] Marsh G. Airframers exploit composites in battle for supremacy[J]. Reinforced Plastics, 2005, 49(3):26-32.

[2] Xue P C, Li H, Chang $Y$ L, et al. Natural frequency calculation and validation of fiber reinforced composite thin plate under cantilever boundary[J]. Journal of Aerospace Power, 2016, 31(7):1754-1760.

[3] Xu Z, Li H, Xue P C, et al. Natural Characteristics Analysis and Validation of Fiber MetalLaminates Thin Plates Under Cantilever Boundary[J]. Journal of Northeastern University (natural science), 2018, 39(12):1737-1742.

[4] Tao J, Li H G, Pan L, et al. Research and development trend of fiber metal laminates[J]. Journal of Nanjing University of Aeronautics \& Astronautics, 2015, 47(5):626-636.

[5] Cao Z Q. Fiber metal laminates and their applications in aircraft structures[J]. Aeronautical Manufacturing Technology, 2006(6):60-62.
[6] Ren Y S, Liu L H. Research progress on structural damping of fiber reinforced composites[J]. Mechanics in Engineering, 2004, 26(1):9-16.

[7] Li H, Sun W, Chang Y L, et al. Time domain test method for nonlinear damping of a fiber-reinforced composite thin plate with amplitude dependence[J]. Journal of Vibration and Shock, 2018, 37(5):169-174.

[8] Li H, Niu Y, Liang X L, et al. Time-domain test method for nonlinear damping of fiber reinforced composite thin plate based on nonlinear squeezing transform[J]. Journal of Aerospace Power, 2018, 33(6):1456-1463.

[9] Modesto T. López-López, Kuzhir P , Bossis G, et al. Preparation of well-dispersed magnetorheological fluids and effect of dispersion on their magnetorheological properties[J]. Rheologica Acta, 2008, 47(7):787-796.

[10] Xiong C, Lv J G, Zhang J Q, et al. Research on the characteristics, mechanism and application of magneto-rheological intelligent materials[C]// National Conference on Composite Materials. 2002.

[11] Wang J X, Meng G. Research advances in magnetorheological elastomers[J]. Journal of Functional Materials, 2006, 37(5):706-709.

[12] Choi W J, Xiong $Y$ P, Shenoi R A. Vibration Characteristics of Sandwich Beams with Steel Skins and Magnetorheological Elastomer Cores[J]. Advances in Structural Engineering, 2010, 26(13):837-844.

[13] Dwivedy S K, Mahendra N, Sahu K C. Parametric instability regions of a soft and magnetorheological elastomer cored sandwich beam[J]. Journal of Sound \& Vibration, 2009, 325(4-5):686-704.

[14] Ni Y Q, Ying Z G, Chen Z H. Magneto-rheological elastomer (MRE) based composite structures for micro-vibration control[J]. Earthquake Engineering and Engineering Vibration, 2010, 9(3):345-356.

[15] Deng HX, Gong XL, Wang LH. Development of an adaptive tuned vibration absorber with magnetorheological elastomer[J]. Smart Materials and Structures, 2006, 15(5):N111-N116.

[16] Wei K X, Meng G, You H, et al. Experimental study on vibration response characteristics of a magnetorheological elastomer sandwich beam[J]. Journal of Vibration and Shock, 2009, 28(10):81-83.

[17] Hu G L, Li B B, Jiang M, et al. Analysis of Vibration Control Performance of Magnetorheological Sandwich Beam Based on MRF and MRE Materials[J]. Machine Tool \& Hydraulics, 2018, 46(11):101-105. 